\title{
Modeling Framework and Coordination of DER and Flexible Loads for Ancillary Service Provision
}

\author{
Jorge García, Juan M. Víquez, José Incer \\ Francisco Escobar, Gustavo Valverde \\ Engineering Research Institute (INII) \\ University of Costa Rica \\ gustavo.valverde@ucr.ac.cr
}

\author{
Petros Aristidou \\ Department of Electrical Engineering, \\ Computer Engineering \& Informatics \\ Cyprus University of Technology \\ petros.aristidou@cut.ac.cy
}

\begin{abstract}
Distributed energy resources and demand-response initiatives are expected to increase the flexibility of future power systems. This paper presents new models of individual photovoltaic systems, batteries, and thermostatically controlled loads that can be used to propose and validate new coordination schemes. Unlike previous works that focus on aggregate representations, these models make it possible to represent more accurately the capabilities and limitations of these resources. For demonstration purposes, the paper proposes a new coordination scheme that employs signals to individual units without having full information of their actual conditions. This low-cost scheme is able to steer the distributed units towards the desired operation. It is expected that mature versions of this type of coordinators will provide new tools and measures for system operators to face abnormal system conditions without high investment costs.
\end{abstract}

\section{Introduction}

Modern power systems are moving towards cleaner and decentralized generators installed at medium- and Low-Voltage (LV) levels. The increase of Distributed Energy Resources (DERs) connected to Distribution Networks (DNs) will change the way power systems are operated. DERs and Flexible Loads (FLs), such as Thermostatically Controlled Loads (TCLs) and Electric Vehicles (EVs), have great potential to participate in voltage and frequency control of power systems due to their high flexibility and controllability. Some grid codes already envision the participation of DERs in voltage support of Transmission Networks (TNs) [1], while demand-response aggregation is already considered as a competitive resource for frequency control [2].

Due to computational constraints and limited availability of data, the impact of small-scale DERs on TNs has been analyzed using aggregate models that capture their overall behavior. Similarly, aggregate models for FLs have been implemented for demand response studies [3]. However, these aggregate models may under- or over-estimate the overall response of the units, since the physical phenomena that define the constraints and time response of individual units are ignored. For example, Air Conditioning (AC) units can operate as FLs because they can temporary interrupt or reduce their demand without significant user discomfort. The precise instants at which they can participate on voltage or frequency control depend on local factors, such as the outside temperature, the desired room temperature, and the thermal characteristics of the room. Furthermore, the type of the AC system, i.e. conventional or inverter-based, dictates its capability to provide services [2]. This behavior and interaction with the grid is often lost in aggregate models.

The multiple services that DERs and FLs can provide require coordination and prioritization of actions, which can only be tested and validated with disaggregated models. This detailed representation of DERs and FLs within large-scale TN-DN system models can facilitate the understanding of how these units can be coordinated to provide services at different voltage levels. For this, the models need to account for internal controllers, unit limitations and time delays.

In this paper, we present a modeling framework for DERs and some FLs for dynamic studies to characterize and assess their ability to provide ancillary services in coordinated control schemes. We introduce the models based on three interacting building blocks: (a) the physical model, (b) the internal controllers as well as those related to voltage and frequency support, and (c) the electric model. Each of these blocks imposes constraints on the available power and energy, and on the time availability to provide grid support.

The models are able to represent the physical 


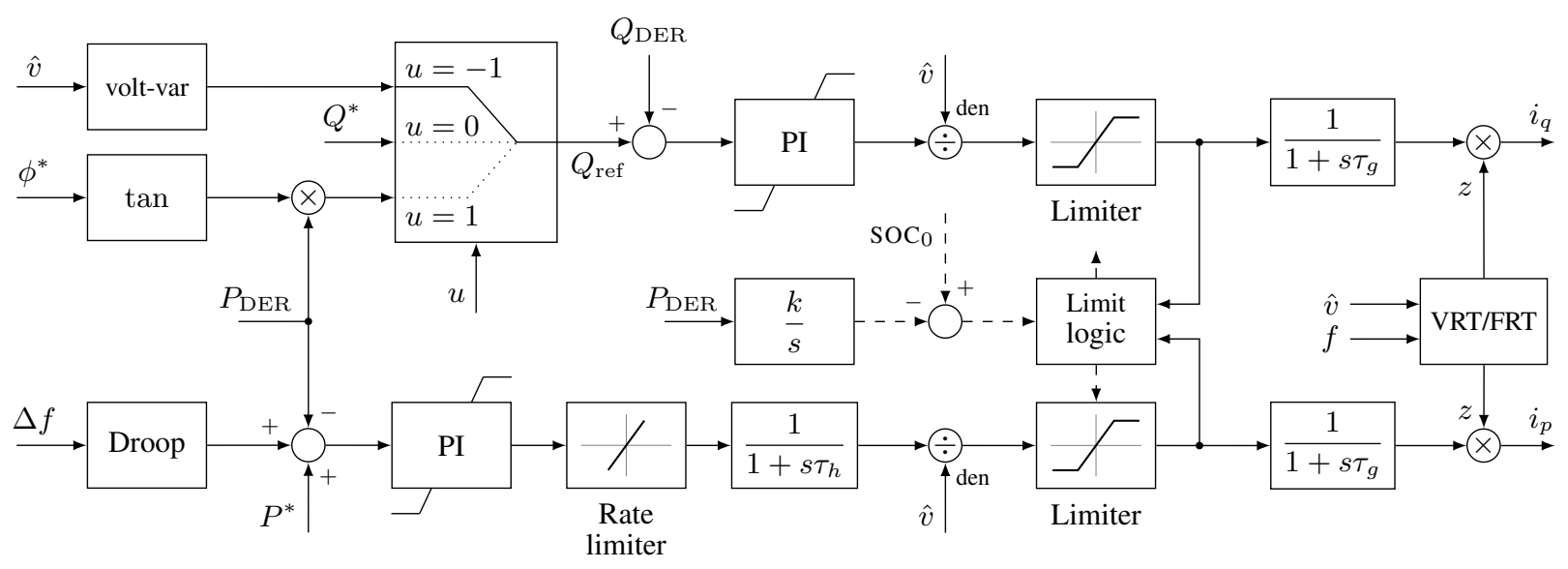

Figure 1. Internal controller of the proposed DER_D model.

phenomena that govern the behavior of each unit - a critical trait for designing controllers for the provision of ancillary services. Although this papers focuses on DERs, water heaters, and $\mathrm{AC}$ units, the concept can be extended to other FLs. Finally, we present a new coordination scheme to steer DERs and FLs for network control.

The remainder of this paper is organized as follows: Section 2 provides a description of the dynamic models and the typical parameters of DERs and FLs. In Section 3 we introduce the controllers that make it possible to coordinate DERs and FLs for service provision. The validation of the models and a proof of concept of a novel coordinator are presented in Section 4. Finally, conclusions are drawn in Section 5.

\section{Individual DER and FL Modeling}

In this section, we present the models of the DERs and some FLs. The latter are related to TCLs, more specifically inverter-interfaced AC units and Electric Water Heaters (EWHs).

\subsection{Inverter-interfaced DERs}

Inverter-interfaced DERs, such as Photovoltaic (PV) Systems and Battery Energy Storage Systems (BESS), have reached important penetration levels in modern power systems. The Western Electricity Coordinating Council (WECC) has developed dynamic models for large-scale and aggregate PV systems and BESS. In particular, the PVD1 [4] and the DER_A [5] are used to represent aggregate DER models. However, they are not suitable for individual units located in LV systems. Hence, a new model called DER_D, derived from DER_A, is proposed in this work.

The new DER_D model, shown in Fig. 1, allows representing disaggregated PVs and BESS at household
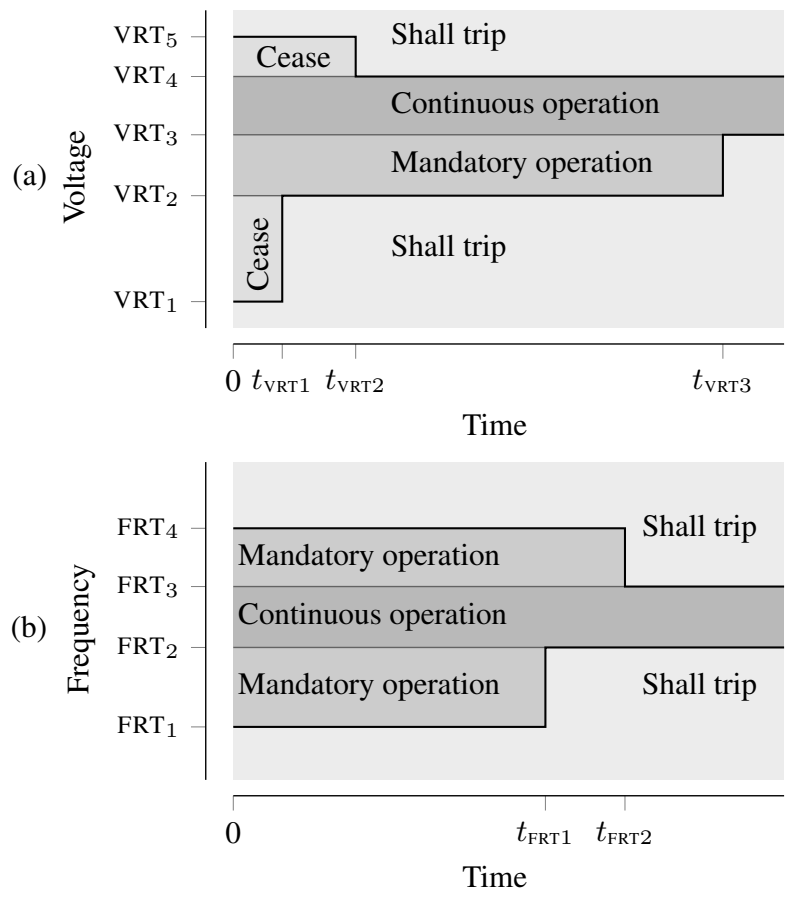

Figure 2. (a) Voltage and (b) frequency ride-through curves [6].

level. The inputs marked with an asterisk represent control setpoints. A BESS can be modeled by the dashed central branch in Fig. 1. The integrator output is used to represent the State-of-Charge (SOC) of the battery during charging, discharging or idle conditions.

Either as PV or BESS, the model contains two control branches with Proportional-Integral (PI) controllers, one for reactive power and the other for active power. The reactive power branch allows to operate in constant reactive power mode (flag $u=0$ ), constant power factor 


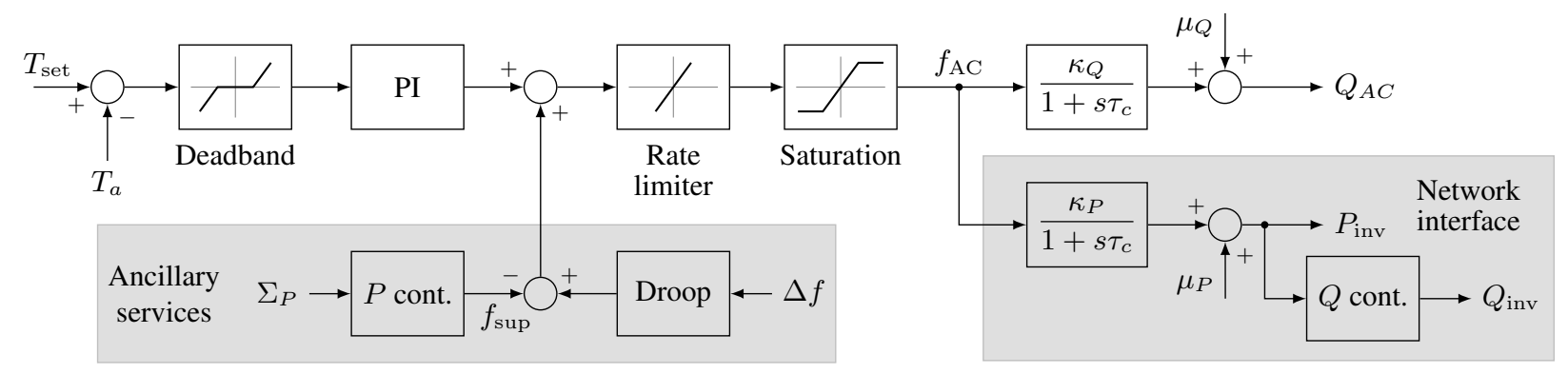

Figure 3. Internal controllers of the inverter-interfaced AC unit.

mode (flag $u=1$ ) or Volt-Var mode (flag $u=-1$ ). Additionally, the active power branch may be used to participate in primary frequency control thanks to the droop block. The model also includes a current-limiting logic and allows to select between active or reactive power priority, similar to DER_A.

Aiming to represent each DER independently, and following the IEEE Std. 1547 [6], DER_D integrates a tripping scheme based on Voltage and Frequency Ride-Through (VRT/FRT) characteristics to define the conditions for which DERs must operate or trip, through the binary multiplier $z$. Unlike the DER_A model, the VRT and FRT curves trip the DER instead of tripping just a fraction, since the variant is intended to represent individual units. For the VRT, Fig. 2(a) shows both the low (LVRT) and high (HVRT) curves. Similarly, Fig. 2(b) shows the low (LFRT) and high (HFRT) curves.

The Continuous Operation region in VRT and FRT represents normal operation. The DER power output $P_{\mathrm{DER}}$ and $Q_{\mathrm{DER}}$, related to $i_{p}$ and $i_{q}$, are regulated by the PI controllers. For the Mandatory Operation region, the DERs shall maintain their output during the disturbance, as long as its duration is not longer than $t_{\mathrm{VRT} 3}$ seconds for the LVRT or $t_{\mathrm{FRT} 1}$ and $t_{\mathrm{FRT} 2}$ seconds for the FRT. Finally, Cease Operation region allows the DERs either to continue exchanging current or to stop injection. This, however, does not mean actual tripping because the DER may be required to recover its power output if normal conditions are restored before $t_{\mathrm{VRT} 1}$ or $t_{\mathrm{VRT} 2}$.

\subsection{Inverter-interfaced AC Units}

Conventional AC units keep the temperature inside a room between two limits $T_{-}$and $T_{+}$. When the temperature falls below $T_{-}$, the AC switches OFF and then switches back oN when the room temperature overpasses $T_{+}$. This behavior results in a cyclic power consumption between $0 \mathrm{~W}$ and the nominal power of the $\mathrm{AC}$ unit. On the contrary, modern $\mathrm{AC}$ units are equipped with inverters that modify the AC compressor frequency according to the temperature requirements. This control makes the AC unit operate continuously at a very low power consumption.

To describe the thermal dynamics, this paper considers a two-mass model that is based on the energy balance [7]. The temperature $T_{a}$ of the air inside the room and the temperature $T_{m}$ of the solid-masses are assumed to evolve according to two differential equations:

$$
\begin{aligned}
C_{a} \frac{d T_{a}}{d t} & =T_{m} H_{m}-T_{a}\left(U_{a}+H_{m}\right)+T_{o} U_{a} \\
& +\left(Q_{i}+Q_{s}\right) / 2-Q_{A C} \\
C_{m} \frac{d T_{m}}{d t} & =H_{m}\left(T_{a}-T_{m}\right)+\left(Q_{i}+Q_{s}\right) / 2
\end{aligned}
$$

Here, $C_{a}$ and $C_{m}$ are the heat capacity of air and of the building materials and furniture, respectively. $T_{o}$ is the outside temperature, $U_{a}$ is the heat transfer coefficient between the inner and outer air mass, and $H_{m}$ is the heat transfer coefficient between the inner air and the solid masses. Moreover, $Q_{i}$ is the heat gain from internal appliances, $Q_{s}$ is heat gain from solar radiation and $Q_{A C}$ is the removed heat by the cooling system.

The thermal subsystem is coupled to the electrical subsystem as depicted in Fig. 3. The compressor frequency $f_{\mathrm{AC}}$ changes if the difference between $T_{a}$ and the desired temperature $T_{\text {set }}=\left(T_{+}-T_{-}\right) / 2$ is large enough to exceed the deadband. A PI controller is used to regulate this frequency. When $T_{a}$ falls outside these limits, the controller modifies the compressor frequency to bring $T_{a}$ within the temperature deadband [2].

The power demand $P_{\text {inv }}$ and the heat $Q_{\mathrm{AC}}$ from the $\mathrm{AC}$ unit are functions of $f_{\mathrm{AC}}$, given in the frequency domain by [2]:

$$
\begin{aligned}
P_{\mathrm{inv}}(s) & =\frac{\kappa_{P}}{1+s \tau_{c}} f_{\mathrm{AC}}(s)+\mu_{P} \\
Q_{\mathrm{AC}}(s) & =\frac{\kappa_{Q}}{1+s \tau_{c}} f_{\mathrm{AC}}(s)+\mu_{Q}
\end{aligned}
$$

where $\kappa_{P}, \kappa_{Q}, \mu_{P}, \mu_{Q}$ are constant coefficients of the unit and $\tau_{c}$ is the time constant of the compressor. 


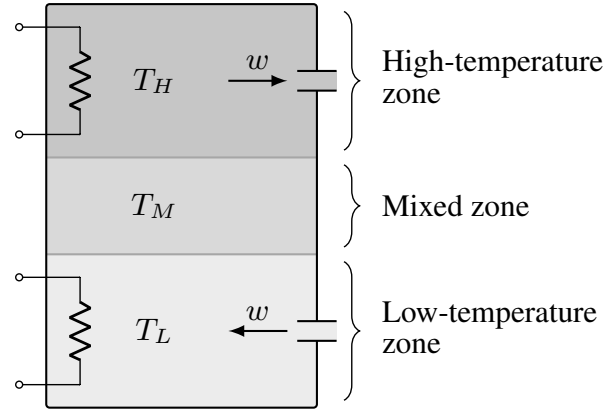

Figure 4. EWH components and temperature zones.

The reactive power output $Q_{\text {inv }}$ depends on the inverter's ability to consume or inject reactive power, according to the actual active power $P_{\text {inv }}$. It is assumed that the inverter can change its reactive power output with a PI controller.

\subsection{Electric Water Heaters}

The EWHs are described using a zone-stratified thermal model [8] that considers the high-, mixed-, and low-temperature zones depicted in Fig. 4. It is assumed that there are two independent heating elements in the tank, one located at the high-temperature zone and the other at the low-temperature zone. Note that the hot water outlet is located at the high-temperature zone while the cold inlet is in the low-temperature zone.

Similar to the case of the AC units, this model associates a single temperature to each region and is based on the energy balance. The high, mixed, and low temperatures, denoted by $T_{H}, T_{M}$, and $T_{L}$, respectively, evolve according to three differential equations:

$$
\begin{aligned}
C_{p H} \frac{d T_{H}}{d t}= & \rho_{W} w c_{p}\left(T_{M}-T_{H}\right)+G_{s c H}\left(T_{o}-T_{H}\right) \\
& +K_{s M} T_{M}-K_{s H} T_{H}+Q_{H} \\
C_{p M} \frac{d T_{M}}{d t}= & \rho_{W} w c_{p}\left(T_{L}-T_{M}\right)+G_{s c M}\left(T_{o}-T_{M}\right) \\
& +K_{s H} T_{H}-2 K_{s M} T_{M}+K_{s L} T_{L} \\
C_{p L} \frac{d T_{L}}{d t}= & \rho_{W} w c_{p}\left(T_{i n}-T_{L}\right)+G_{s c L}\left(T_{o}-T_{L}\right) \\
& +K_{s M} T_{M}-K_{s L} T_{L}+Q_{L}
\end{aligned}
$$

where $C_{p X}$ is the heat capacity of the high $(X=H)$, mixed $(X=M)$ and low $(X=L)$ zones, $\rho_{W}$ is the mass density of water and $c_{p}$ is the specific heat of water. The coefficient $G_{s c X}$ represents the losses between each zone and the surroundings, which depend on the thermal resistance and tank insulation. $K_{s X}$ is the conductivity coefficient in each zone, which defines the heat exchange between zones, and $Q_{X}$ is the heat produced by the heating elements located in the high- and low-temp. $T_{o}$ is the ambient temperature and $T_{i n}$ is the inlet water temperature. Finally, $w$ is the water flow rate.

From the electric point of view, this EWH is modelled by two identical resistors with power $P_{\mathrm{WH}}$. The water temperature of the high and low zones are controlled by thermostats (with hysteresis) following a master-slave scheme. Here, only one of the heating elements is ON at a time. When both temperatures are outside their corresponding limits, the upper resistor will have priority. The following equations describe this control:

$$
\begin{aligned}
& P_{H}= \begin{cases}P_{\mathrm{WH}}, & \text { if } T_{H}<T_{-} \\
0, & \text { if } T_{H}>T_{+}\end{cases} \\
& P_{L}=\left\{\begin{array}{ll}
P_{\mathrm{WH}}, & \text { if } T_{L}<T_{-}^{\prime} \\
0, & \text { otherwise }
\end{array} P_{H}=0\right.
\end{aligned}
$$

where $P_{H}$ (resp. $\left.P_{L}\right)$ and $T_{-}$(resp. $T_{-}^{\prime}$ ) are the resistor power demand and the minimum allowed temperature at the high-temperature (resp. low) zone. Similarly, $T_{+}$is the maximum allowed temperature for both zones. The actual power demand $P_{\mathrm{WH}}$ of the EWH will depend on the resistors and the applied voltage.

\section{Coordination of DERs and FLs}

A novel scheme is proposed in this paper to coordinate the FLs and DERs. The coordinator is able to send signals to the individual units aiming at steering their response, without knowing the actual condition of the downstream units and how many will actually respond. For simplification, it is assumed that the signals $\Sigma$ are numbered from one to five according to the level of support required by the coordinator, where one is kept when no participation is needed and five is sent when maximum participation of DERs and loads is needed. The way each DER and FL respond to the coordinator requests is explained in the following subsections.

\subsection{Inverter-interfaced DERs}

PV systems and BESS will consider two algorithms: one related with reactive power $(\mathrm{Q})$ support and another related with active power $(\mathrm{P})$ support. When the active power output of these elements is modified to provide support to the system, there is a direct affectation to the user. Considering this, the control proposed will firstly request these units to provide $\mathrm{Q}$ support. $\mathrm{P}$ support will only be requested if the system is still at risk and therefore, system security is prioritized over user affectation.

Regarding the Q support scheme, the proposed 
DER_D model is adapted to include an additional control logic to receive and react to external signals. In short, the additional control consists of a modified Volt-Var curve which allows the DER to follow or ignore external requests according to terminal voltage $\hat{v}$. The details of the logic is presented in [1]. This was integrated in DER_D directly in the Volt-Var block, activated with $u=-1$. The reactive power support depends on four aspects:

- Request direction: The coordinator may ask the DER to inject or consume $\mathrm{Q}$, depending on what is needed at higher voltage levels. When no support is required, the DER will follow its predefined Volt-Var curve.

- Participation region: the Volt-Var curve considers a region $v_{2} \leq \hat{v} \leq v_{3}$ for which the DERs can follow thecoordinator, as shown in Fig. 5. Outside of this region, the DERs will ignore the requests and follow the original Volt-Var curve.

- $t_{\text {sample }}$ : the reference value $Q_{\text {ref }}$ that the PI controller receives at instant $t_{k}$ is updated with a period of $t_{\text {sample }}=$ $t_{k}-t_{k-1}$. The lower the value of $t_{\text {sample }}$, the faster the help is provided by the DER.

- Helping fraction $(\rho)$ : the increment of help of DERs between requests depends on the parameter $\rho$, which defines the maximum allowed $\Delta Q_{\text {ref }}$ as percentage of the maximum (resp. minimum) reactive power $Q_{\max }$ (resp. $\left.Q_{\min }\right)$.

The reference of the DER when the coordinator requests to inject $\left(\Sigma_{Q}>0\right)$ reactive power at instant $t_{k}$ is [1]:

$$
Q_{\text {ref }}\left[t_{k}\right]=Q_{\text {ref }}\left[t_{k-1}\right]+\rho \frac{v_{3}-\hat{v}}{v_{3}-v_{2}} Q_{\max }\left[t_{k}\right]
$$

where $Q_{\text {ref }}\left[t_{k-1}\right]$ is the power reference at $t_{k-1}$. In the case of commands to consume $\left(\Sigma_{Q}<0\right)$ reactive power:

$$
Q_{\mathrm{ref}}\left[t_{k}\right]=Q_{\mathrm{ref}}\left[t_{k-1}\right]+\rho\left(1-\frac{v_{3}-\hat{v}}{v_{3}-v_{2}}\right) Q_{\mathrm{min}}\left[t_{k}\right]
$$

where $Q_{\min }=-Q_{\max }$.

During the participation of DERs, the values of $t_{\text {sample }}$ and $\rho$ may be updated depending on the severity of the problem seen by the coordinator. These values follow the piecewise functions presented in Fig. 6. The higher participation is required, the greater the $\rho$ value and the lower the $t_{\text {sample }}$.

As shown in Fig. 5, the region in which the DERs can follow the coordinator requests is restricted. However, depending on the severity of the problem, the DERs must prioritize system security over local voltage problems. Therefore, when $\left|\Sigma_{Q}\right|$ exceeds the threshold $\Sigma Q_{\text {exp }}$, the participation region is widened.

P support scheme is intended to help the system

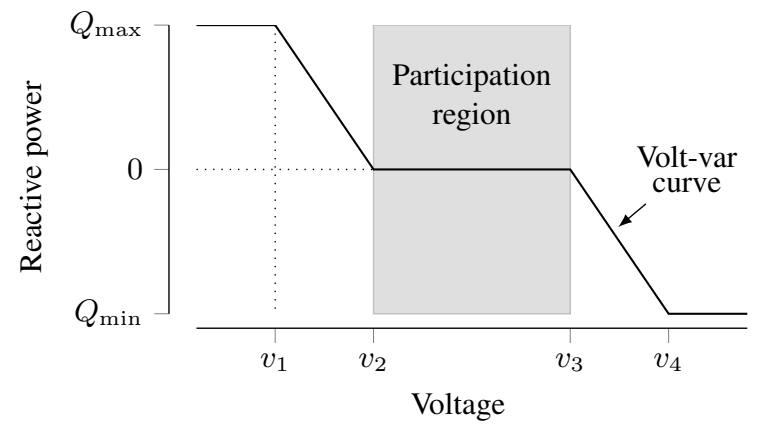

Figure 5. Participation region in the VQ-plane of the proposed DER_D model.

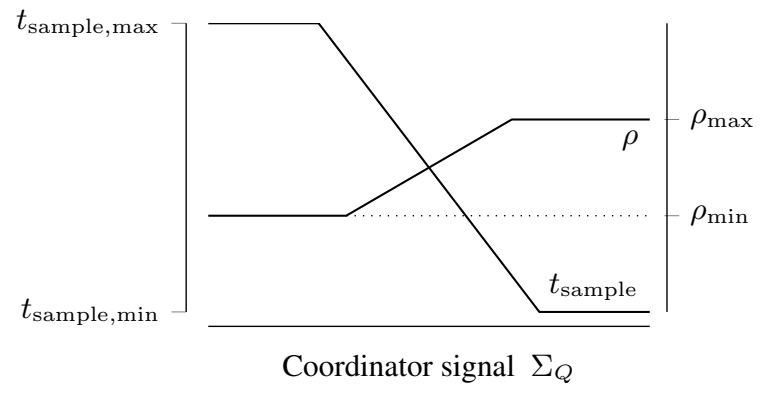

Figure 6. Piecewise functions for $t_{\text {sample }}$ and $\rho$.

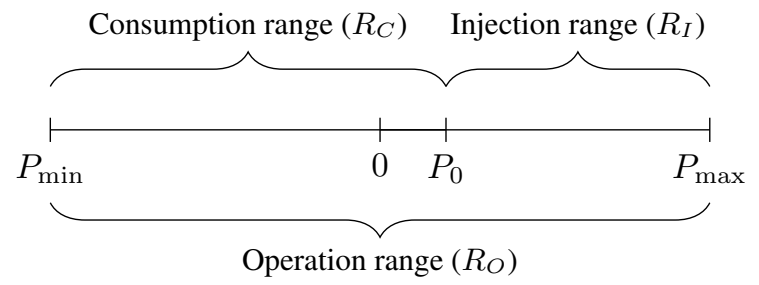

Figure 7. Battery active power availability ranges (for a PV, $P_{\min }=0$ ).

when severe conditions occur $\left(\left|\Sigma_{P}\right|=4 / 5\right)$ and no more $\mathrm{Q}$ support can be provided. During these situations, DERs active power output is given by $P_{\mathrm{DER}}=P_{o}+$ $\Delta P_{\mathrm{DER}}$, where $P_{o}$ is the initial value and $\Delta P_{\mathrm{DER}}$ is calculated based on the active power availability ranges depicted in Fig. 7:

$$
\Delta P_{\mathrm{DER}}= \begin{cases}-\left|R_{\mathrm{C}}\right| \times F_{4} & \text { if } \Sigma_{P}=-4 \\ -\left|R_{\mathrm{C}}\right| \times F_{5} & \text { if } \Sigma_{P}=-5 \\ \left|R_{\mathrm{I}}\right| \times F_{4} & \text { if } \Sigma_{P}=4 \\ \left|R_{\mathrm{I}}\right| \times F_{5} & \text { if } \Sigma_{P}=5\end{cases}
$$

$F_{4}$ and $F_{5}$ are positive fractions that determine the displacement that $P$ will have in the consumption/injection range. Since greater support is 


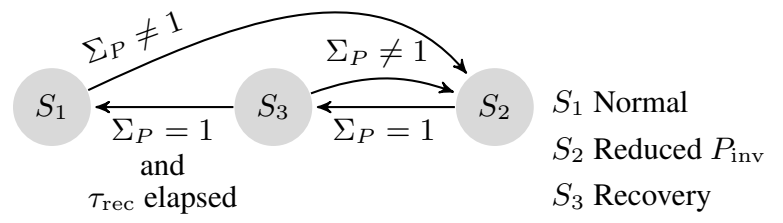

Figure 8. Active-power control modes of inverted-interfaced $A C$ units.

required during $\left|\Sigma_{P}\right|=5$, these two fractions should satisfy $F_{4}<F_{5}$. For this purpose, $F_{4}=0.5$ and $F_{5}=1.0$ were selected. It is important to say that PVs can only obey a request if $\Sigma_{P}<0$, since they can not increase their output as they depend of the solar radiation. Once $\left|\Sigma_{P}\right|<4$, the active power output returns to $P_{o}$.

\subsection{Inverter-interfaced AC Units}

The ancillary services provided by the AC unit are carried out through a reduction of active power $\left(P_{\text {inv }}\right)$ consumption and variation of reactive power production/consumption ( $\left.Q_{\text {inv }}\right)$. Their control is independent of each other based on the signal sent from the coordinator, i.e. $\Sigma_{P}$ and $\Sigma_{Q}$. This means that $Q_{\text {inv }}$ changes can be done while keeping $P_{\text {inv }}$ constant to satisfy a specific room temperature. Still, the power output of the inverter will always be limited by the nominal apparent power $\left(S_{\text {nom }}\right)$ of the AC unit.

Active Power Control: $P_{\mathrm{AC}}$, and thus $P_{\text {inv }}$, has a linear relation with $f_{\mathrm{AC}}$ as defined in (2). Since $f_{\mathrm{AC}}$ is limited by $f_{\max }$ and $f_{\min }, P_{\text {inv }}$ is limited accordingly. To provide ancillary services, the AC unit must reduce its power consumption through $f_{\mathrm{AC}}$ reductions. For the active power control, four stages are proposed: normal operation $\left(\Sigma_{P}=1,2\right.$ or 3$)$, support $\left(\Sigma_{P}=4\right)$, emergency $\left(\Sigma_{P}=5\right)$ and recovery. These stages are shown with a Finite State Automaton (FSA) in Fig. 8:

-Normal operation (state $S_{1}$ ): In normal conditions when $\Sigma_{P}=1$, the AC unit will operate with the $P_{\text {inv }}$ and $f_{\mathrm{AC}}$ that keep the room temperature $T_{a}$ close to $T_{\text {set }}$. As no support is required, $f_{\text {sup }}$ is 0 in Fig. 3.

-Support Stage (state $S_{2}$ ): Under this condition, the power consumption of the AC unit will be reduced through $f_{\mathrm{AC}}$ by a factor $f_{\text {frac }}$. In this case, $f_{\text {sup }}$ is defined by:

$$
f_{\text {sup }}=f_{\text {frac }}\left(f_{\text {inv }}-f_{\text {min }}\right)
$$

where $f_{\text {frac }}$ is arbitrary chosen by the user, e.g. 0.5.

-Emergency (state $S_{2}$ ): This is the worst scenario and the AC unit must be driven to its minimal power consumption. This means $f_{\text {sup }}=f_{\min }$.
Table 1. $Q_{\text {factor }}$ as a function of $\Sigma_{P}$ and $\Sigma_{Q}$.

\begin{tabular}{lcc}
\hline$\Sigma_{P}$ & $\Sigma_{Q}$ & $Q_{\text {factor }}$ \\
\hline $1,2,3$ & 1 & -0.1 \\
$1,2,3$ & 2 & 0.25 \\
$1,2,3$ & 3 & 0.75 \\
$1,2,3$ & 4,5 & 1 \\
4,5 & - & 1 \\
\hline
\end{tabular}

-Recovery (state $S_{3}$ ): This is needed to avoid undesired synchronization of the recovery of multiple $\mathrm{AC}$ units. For each $\mathrm{AC}$ unit, a recovery time $\tau_{r e c}$, in minutes, is randomly assigned. Therefore, when moving from $\Sigma_{P}=5$ to $\Sigma_{P}=1,2$ or 3 , the AC unit will take $\tau_{r e c}$ minutes to go back to normal operation.

Reactive Power Control: The AC unit is able to inject or consume reactive power as requested by the $\Sigma_{Q}$ signal. The available reactive power is locally computed from $S_{\text {nom }}$ and $P_{\text {inv }}$ :

$$
Q_{\text {available }}=\sqrt{{S_{\text {nom }}^{2}-P_{\text {inv }}^{2}}^{2}}
$$

Under normal conditions $\left(\Sigma_{Q}=1\right)$, it is assumed that the AC unit consumes $Q_{\text {inv }}$ based on $P_{\text {inv }}$ and the actual lagging power factor. When the coordinator requests support, i.e. $\Sigma_{Q}=2,3,4$ or 5 , the AC unit injects reactive power. This injection is calculated based on $Q_{\text {available }}$ and the $Q_{\text {factor }}$. The latter is defined in Table 1 . The minus sign for $Q_{\text {factor }}$ with $\Sigma_{Q}=1$ indicates that the AC unit was consuming $Q$. When $\Sigma_{P}=4$ or 5, the maximum $Q$ will be requested, i.e. $Q_{\text {factor }}=1$. Based on this, the new $Q_{\text {ref }}$ for the AC unit is defined by:

$$
Q_{\text {ref }}=Q_{\text {available }} \cdot Q_{\text {factor }}
$$

To achieve the desired $Q_{\text {ref }}$, an internal PI controller eliminates the control error between $Q_{\text {inv }}$ and $Q_{\text {ref }}$. The $Q_{\text {cont }}$ block shown in Fig. 3 includes the reactive power control described before.

\subsection{Electric Water Heaters}

Some control strategies for large populations of TCLs either act individually on each unit [9] or prioritize which units should be switched ON or OFF based on their temperatures [10]. However, this is not feasible in the present scheme, since an undifferentiated signal $\Sigma$ is sent to all the FLs and the coordinator ignores the thermal dynamics of the available TCLs. We propose to control the power consumption of each TCL by changing its working time by a factor $\gamma \in[0,1]$. The working time of a TCL is defined here as its average ON time per cycle over several cycles.

During normal operation, with state $S_{1}$, the average working time $\tau_{\mathrm{ON}}$ and the average idle time $\tau_{\mathrm{OFF}}$ are used. 


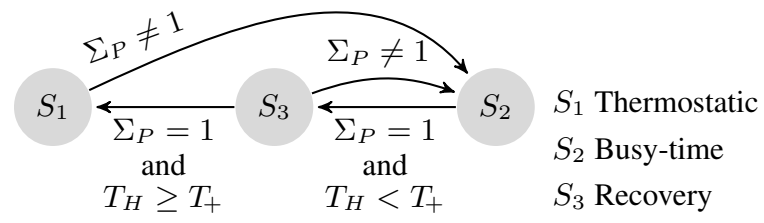

Figure 9. Control modes of EWHs.

These values are computed locally by the EWH using the average period $\tau$ and the duty cycle $\eta$. Although the switching due to the two thermostats is not perfectly cyclic, and thus $\tau$ and $\eta$ are not uniquely defined, they can be approximated by measuring the ON and OFF times per cycle over several cycles. Given $\eta$ and $\tau$, variables $\tau_{\mathrm{ON}}$ and $\tau_{\mathrm{OFF}}$ are calculated as follows:

$$
\tau_{\mathrm{ON}}=\eta \tau, \quad \tau_{\mathrm{OFF}}=(1-\eta \tau)
$$

Upon detecting $\Sigma_{P} \neq 1$, the EWH stops following its thermostat and starts behaving as a timed automaton, as presented in Fig. 9. In State $S_{2}$, an internal timer forces the unit to stay ON no longer than $\gamma \tau_{\mathrm{ON}}$, and to stay OFF no longer than $\tau-\gamma \tau_{\mathrm{ON}}$. In presence of two thermostats, this can be accomplished by blocking the top heating element and controlling the bottom heating element alone, which is responsible for most of the power consumption. Higher values of $\Sigma_{P}$ result in smaller values of $\gamma$, and the total active power demanded by the EWHs will be decreased by the same factor.

If all the EWHs turn ON immediately after $\Sigma_{P}$ switches back to 1 , undesired synchronization of the units will likely occur, which may result in counterproductive effects for the grid. To avoid this, a recovery state $S_{3}$ is used. Within this state, the EWH will remain ON (resp. OFF) no longer than $k \tau_{\mathrm{ON}}$ (resp. $\tau-k \tau_{\mathrm{ON}}$ ), where $k$ is calculated based on the current value of $T_{H}$ and is higher than 1 . The lower the value of $T_{H}$, the higher $k$. The EWH will return to $S_{1}$ once $T_{H}>T_{+}$.

In this paper, the participation of EHWs will only be considered for $\Sigma_{P}$ values larger than 2 .

\section{Numerical Results}

To demonstrate the response of both DERs and FLs to the coordinator requests, two types of simulations are considered: support given by AC and EWH populations, and combined support by DERS and FLs in LV networks. In both cases, it is assumed that the coordinator sends the signals without knowing the actual conditions of the DERs and the FLs. As explained in Section 3, the units respond to the coordinator requests according to local decisions. Since these models are suitable for stability studies, only balanced three-phase systems are considered.

The small-scale PV systems and BESS are represented by the proposed DER_D model. DER nominal powers range from $2 \mathrm{~kW}$ to $10 \mathrm{~kW}$. For simplification purposes, the $k$ constant in Fig. 1 is such that all the BESS are able to deliver their nominal power for one hour to go from maximum to minimum SOC.

The EWHs have nominal powers of $1.5 \mathrm{~kW}, 2 \mathrm{~kW}$, $2.5 \mathrm{~kW}$ and $3 \mathrm{~kW}$. For the AC units, their nominal powers range from $0.35 \mathrm{~kW}$ to $1.25 \mathrm{~kW}$ with initial power factors between 1 and 0.95 lagging.

\subsection{Support by AC and EWH populations}

TCLs such as the AC unit and the EWH include slow dynamics that cannot be fully seen in short-term simulations, particularly the recovery period. To show the full response of these loads to external requests, two independent simulations were carried out in RAMSES [11]. A two-bus test system is used for these simulations where the FLs receive the signals from the coordinator at different time instants. The way how this coordinator decides the signal selection is beyond the scope of this paper, and this will be part of a future piece of work. Two scenarios are presented as follows:

Participation of EWHs: 2000 EWHs were connected to the load bus. A 20-hours period was analyzed for different $\Sigma_{P}$ signals. To verify the power demand reduction from these units, the following signal sequence was sent: $\left(\Sigma_{P}, t_{s}\right):(2,3600),(3,9000),(4,14400),(5$, $19800)$ and $(1,20100)$ with $t_{s}$ in seconds. Figure 10(a) shows the EWHs power demand. In this simulation, the EWHs responded for all the $\Sigma_{P}$ values. In this case, $\gamma$ factors of $0.75,0.5$ and 0.25 were considered for $\Sigma_{P}=2, \Sigma_{P}=3$ and $\Sigma_{P}=4$, respectively. It can be observed in Fig. 10(a) that the power demand is reduced at $t=3,600 \mathrm{~s}, t=9,000 \mathrm{~s}$ and $t=14,400 \mathrm{~s}$ by $25 \%$, $50 \%$ and $75 \%$, respectively. At $t=19,800 \mathrm{~s}$, the highest support signal is sent by the coordinator. Under this condition, $\gamma$ factor falls to 0.1, i.e. each EWH demands almost $0 \mathrm{~kW}$.

The recovery process starts at $t=20,100 \mathrm{~s}$ when $\Sigma_{P}$ is set back to 1 . This extreme change from $\Sigma_{P}=5$ to 1 is presented to validate the proposed recovery algorithm. As shown in Fig. 10(a), the EWHs recover their original demand without any evident synchronization.

When the EWHs respond to the coordinator requests, the $T_{H}$ temperatures get decreased, leaving their normal operation limits ( $T_{+}$and $T_{-}$), as can be seen in Fig.10(b). Here, the $T_{H}$ of each EWH was normalized between $T_{+}$and $T_{-}$. This long-term violation of the desired temperature is caused because the coordinator requested 


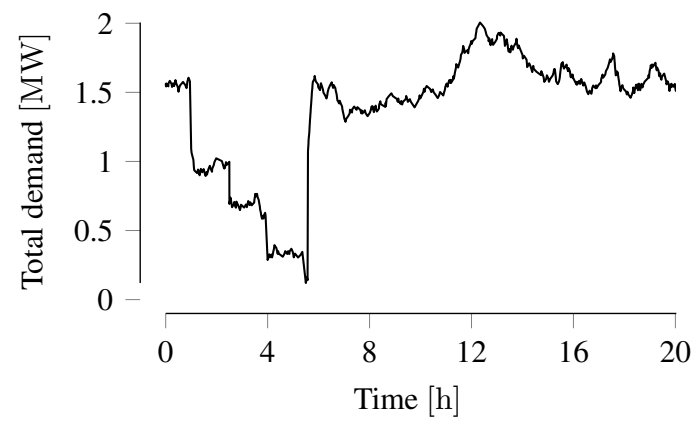

(a)

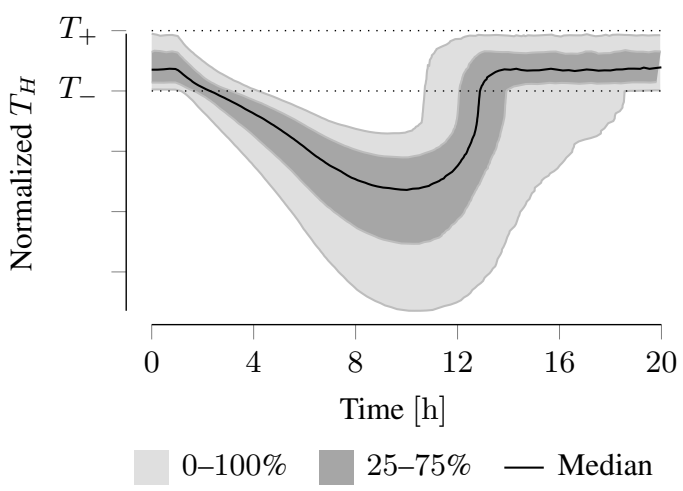

(b)

Figure 10. (a) Total demand of 2000 EWH (b) Normalized $T_{H}$ temperatures. The ticks of the vertical axis are one deadband apart.

up to 4 hours of support.

Participation of Inverter-interfaced AC Units: 1000 AC units were connected to the load bus. The signal sequence sent to all the units in this scenario is $\left(\Sigma_{P} / \Sigma_{Q}\right.$, $\left.t_{s}\right)$ : $(1 / 2,15),(1 / 3,30),(1 / 4,60),(1 / 5,75),(4 / 4,100)$, $(5 / 5,115),(1 / 4,130),(1 / 3,200)$ and $(1 / 1,500)$, with $t_{s}$ in seconds. Fig. 11(a) shows the independent control of $Q$ and $P$ for the first 75 seconds, where only reactive power injection was requested.

The most important aspect is the smooth recovery process when the coordinator switches back to normal operation $(1 / 1,500)$. Despite that the signal was sent at $t=500 \mathrm{~s}$, the powers demanded by the AC units recover their initial condition at about $t=10 \mathrm{~min}$. This was possible through the $\tau_{r e c}$ values randomly selected for each unit in the recovery method. A range of 5 to 10 minutes was used for this purpose. Fig. 11(b) shows the normalized $T_{a}$ temperatures. As the time window for this simulation is in the order of 15 minutes, the $T_{a}$ temperatures do not vary significantly. Depending on the AC unit size and the household, for extended support requests, $T_{a}$ can be significantly affected causing the user discomfort. Economic compensation to the end-user

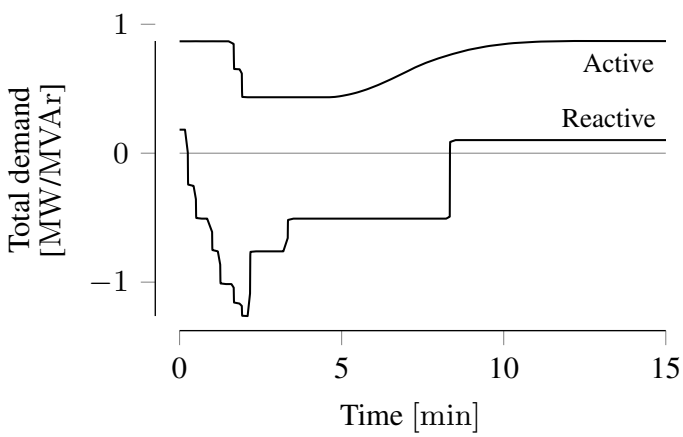

(a)

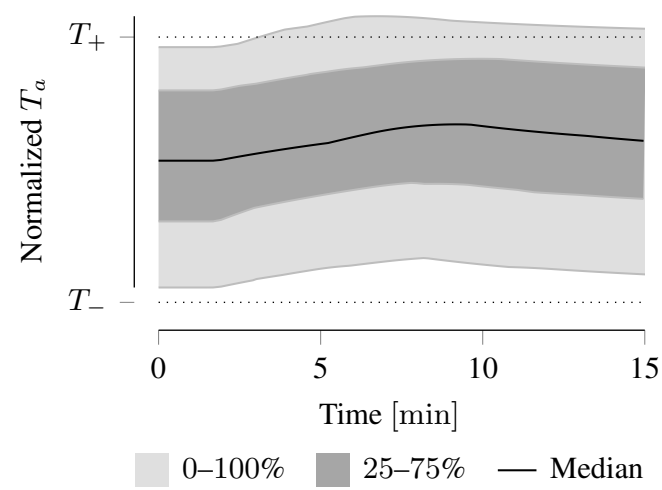

(b)

Figure 11. (a) Total demand of 1000 AC unit (b) Normalized $T_{a}$ temperatures.

should be established.

\subsection{Combined Support of DERs and FLs}

To demonstrate the functionality of the proposed control schemes with multiple DERs (PV systems and BESS) and FLs, a small-scale representative test system was used. Figure 12 presents the one-line diagram for this system. Due to space limitations, the complete LV system is not presented. This LV system, with 131 buses, was populated with DERs and FLs with the methodology described in [12]. In total, there are 62 load buses, 36 DERs, 36 EHWs and 18 AC units.

The signal sequence sent to all the units in this simulation is $\left(\Sigma_{P} / \Sigma_{Q}, t_{s}[\mathrm{~s}]\right):(3 / 3,10),(4 / 4,50)$, $(5 / 5,80),(3 / 3,120),(2 / 2,170)$ and $(1 / 1,210)$.

Figure 13 presents the active and reactive power demand supplied by the transformer, the demand of flexible and non-flexible loads, and the DERs. After the first signal at $t=10 \mathrm{~s}$, all the DERs and FLs contribute to the coordinator either by reducing its power consumption or injecting reactive power to the network. The aggregate response of these units is shown in the active and reactive power supplied by the MV/LV transformer. Note that the 


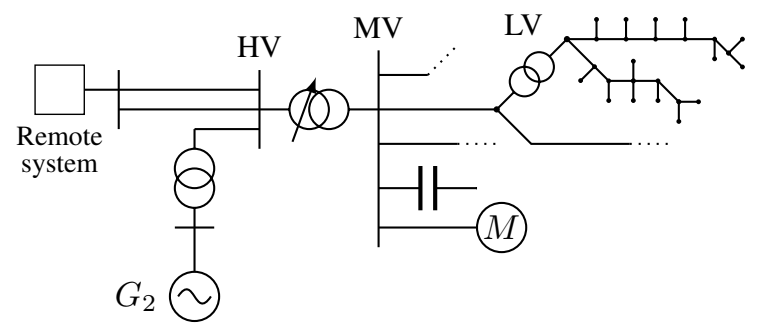

Figure 12. TN-DN test system. The MV and LV topologies are representative.

LV system reduces its active power demand and injects reactive power to the transformer.

Figure 14 presents the evolution of the LV bus voltages due to the coordinator requests. Note that the change in voltages results in small variations in the demand of the voltage-dependent non-flexible loads.

With respect to the DERs, the signal forces changes in $t_{\text {sample }}$ from its maximum value of $5 \mathrm{~s}$ to its minimum value of $1 \mathrm{~s}$. Also, it forces $\rho$ changes from 0 to its maximum value of 0.3 . These changes are reflected on the reactive power injection of the DERs. In these simulations, the threshold $\Sigma Q_{\exp }$ was set to 3 . Therefore, an expansion of the participation region is mandated at $t=10 \mathrm{~s}$. This ensures that all DERs will help the coordinator regardless of their terminal voltage.

For $\Sigma_{Q}=4,5$, and 3 at $t_{s}=50,80$ and $120 \mathrm{~s}$ respectively, the parameters $t_{\text {sample }}$ and $\rho$ remain unchanged at their limiting values. As soon as DERs reach their maximum reactive power capacity, their outputs becomes constant, as shown in Fig. 13(b). Finally, when $\Sigma_{Q}$ returns to 1 at $t_{s}=210 \mathrm{~s}$ (or $3.5 \mathrm{~min}$ ), the DERs intentionally keep their output constant for about $10 \mathrm{~s}$ before restoring to the normal Volt-Var control [1]. This last step takes another $6 \mathrm{~s}$, this is why all DERs have returned to the Volt-var curve at approximately $4 \mathrm{~min}$.

Figure 15 presents the behavior of a specific DER in the VQ-plane. As the time is an implicit variable in this plot, the arrows were added to track its advance. Note that the DER was originally following the Volt-Var curve, and then it started to increase its reactive power output as requested by the coordinator until reaching $Q_{\max }$. Later, when the coordinator stops requesting participation, i.e. $\Sigma_{Q}=1$, the DER finally restores to its Volt-Var curve.

The aggregated active power response of the DERs can be seen in Fig. 13(a) after $t_{s}=50 \mathrm{~s}$, when $\Sigma_{P}$ is raised to 4 . At this point, the DERs start injecting active power to the system based on their individual available power ranges and following (6). At $t_{s}=80 \mathrm{~s}$, when the maximum support is required $\left(\Sigma_{P}=5\right)$, the total active power seen from the transformer becomes negative, indicating that the $\mathrm{LV}$ network is providing active power

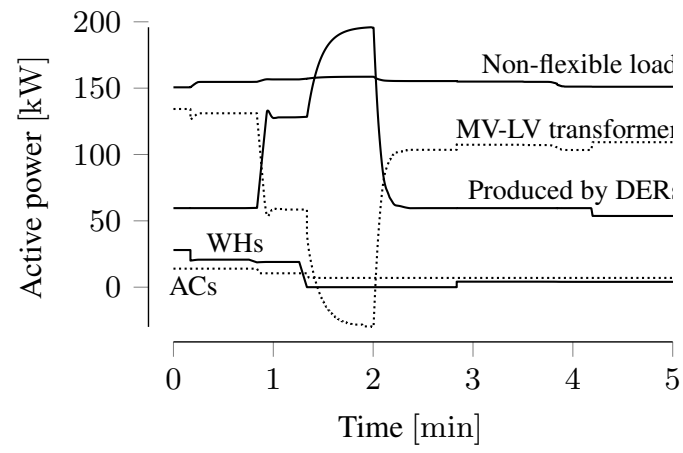

(a)

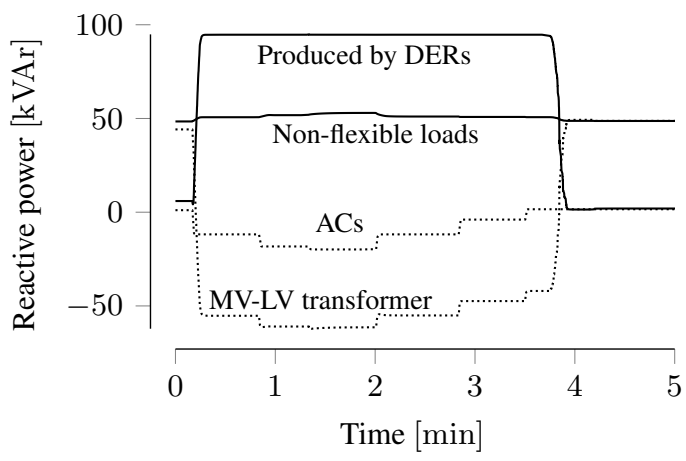

(b)

Figure 13. Active and reactive powers in LV system

to the system. This contribution given by the DERs demonstrate how LV networks with high penetration of DERs, can be coordinated to help power systems.

From the AC units point of view, they initially consume $Q$, i.e. positive values in Fig. 13(a). At $t=10 \mathrm{~s}$, they start injecting reactive power. The contribution is computed using (8), (9) and the factors in Table 1.

The next two signals (at $t=50$ and $80 \mathrm{~s}$ ) consider a change of the AC units power demand. For $\Sigma_{P}=4$, a $f_{\text {frac }}$ of 0.5 was used. During the emergency state when $\Sigma_{P}=5$ at $t=80 \mathrm{~s}$, the AC units consume the minimum active power, whereas the reactive power injection will be maximized (this also occurs with $\Sigma_{P}=4$ ), yet limited by (8). This reactive power response from AC units may not be possible with some inverters.

To recover the original reactive power output, a smooth transition signal was sent by the coordinator at $t=120 \mathrm{~s}, t=170 \mathrm{~s}$ and $t=210 \mathrm{~s}$. As can be seen in Fig. 13(a), the active power demand of the AC units does not change after $t=120 \mathrm{~s}$. As the AC unit were randomly assigned with $5 \leq \tau_{r e c} \leq 15 \mathrm{~min}$, the power recovery of these loads are not observed in this short-term simulation (7 min). However, the recovery method was successfully validated in the long-term simulation in Fig. 11(a). On the other hand, the reactive power output 


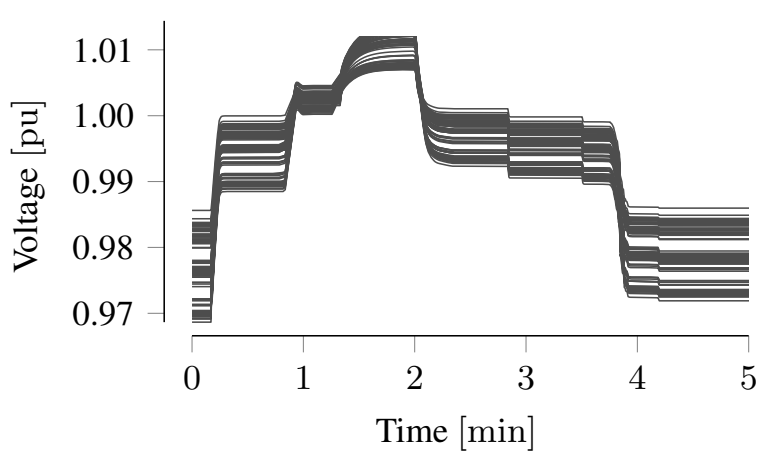

Figure 14. Bus voltages in the LV network.

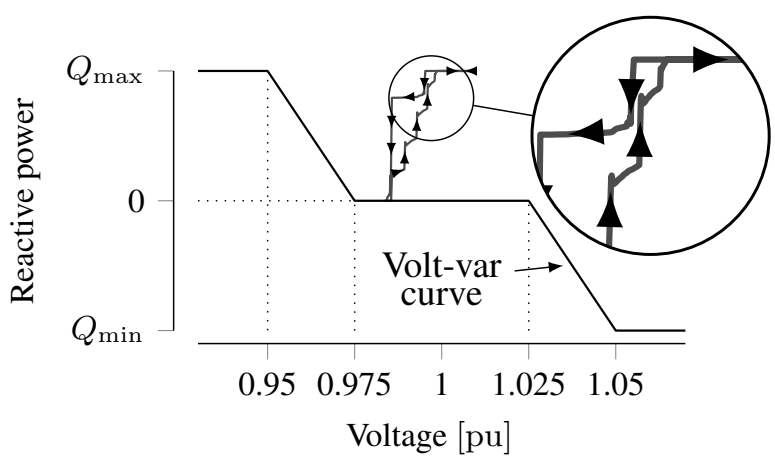

Figure 15. DER output in VQ-plane.

of the AC units moves back to consumption at $t=210 \mathrm{~s}$ (3.5 $\mathrm{min})$, as it was assumed for the initial conditions. This behavior is observed in Fig. 13(b).

Finally, the participation of the EWHs can be seen in Fig. 13(a) through their active power reduction with $\Sigma_{P} \geq 3$, starting at $t=10 \mathrm{~s}$. Reducing the working time $\left(\tau_{\mathrm{ON}}\right)$ by $\gamma$ factor produces a proportional reduction in the active power demand of these units. For this simulation, $\gamma$ values of $0.8\left(\Sigma_{P}=3\right)$ and $0.6\left(\Sigma_{P}=4\right)$ were selected for the EWHs. At $t=80 \mathrm{~s}$, the coordinator requests maximum support with $\Sigma_{P}=5$ so that most of the EWHs are turned OFF until $t=120 \mathrm{~s}(t=2 \mathrm{~min})$ when $\Sigma_{P}=3$. The recovery of these units is not fully presented in the simulation because they have to complete a $\tau_{\text {OFF }}$ period, which can be much larger than $7 \mathrm{~min}$. The recovery process of the EWHs was presented in the long-term simulations.

\section{Conclusion}

Modern power systems will rely more and more on LV-connected DERs and FLs to provide valuable flexibility to the system, and alleviate security problems. In this work, we address modeling requirements of dispersed units and analyze the dynamics of a coordinated scheme aiming to provide ancillary services with these units. More specifically, we propose a modeling platform able to capture the individual behavior and dynamics of DERs and FLs. To assess the performance of the platform, we propose a coordinated control scheme employed on a simplified and a large scale system. The simulation results show that it is possible to steer LV-connected DERs and FLs to participate in network control. Future work will focus on extending the capabilities of the coordinator and evaluating communication delays.

\section{References}

[1] G. Valverde, D. Shchetinin, and G. Hug-Glanzmann, "Coordination of distributed reactive power sources for voltage support of transmission networks," IEEE Transactions on Sustainable Energy, vol. 10, no. 3, pp. 1544-1553, 2019.

[2] H. Hui, Y. Ding, and M. Zheng, "Equivalent modeling of inverter air conditioners for providing frequency regulation service," IEEE Transactions on Industrial Electronics, vol. 66, pp. 1413-1423, Feb 2019.

[3] H. Hao, B. Sanandaji, K. Poolla, and T. Vincent, "Aggregate flexibility of thermostatically controlled loads," IEEE Transactions on Power Systems, vol. 30, 092014.

[4] Western Electricity Coordinating Council (WECC), "Generic solar photovoltaic system dynamic simulation model specification,” tech. rep., WECC, 2012.

[5] North American Electric Reliability Corporation (NERC), "Reliability Guideline: Parameterization of the DER_A Model," tech. rep., NERC, 2019.

[6] "IEEE standard for interconnection and interoperability of distributed energy resources with associated electric power systems interfaces," IEEE Std 1547-2018 (Revision of IEEE Std 1547-2003), pp. 1-138, 2018.

[7] W. Zhang, J. Lian, C. Chang, and K. Kalsi, "Aggregated modeling and control of air conditioning loads for demand response," IEEE transactions on power systems, vol. 28, no. 4, pp. 4655-4664, 2013.

[8] M. Zuñiga, K. Agbossou, A. Cardenas, and L. Boulon, "Parameter estimation of electric water heater models using extended kalman filter," in IECON 2017 - 43rd Annual Conference of the IEEE Industrial Electronics Society, pp. 386-391, 2017.

[9] M. Liu and Y. Shi, "Model predictive control of aggregated heterogeneous second-order thermostatically controlled loads for ancillary services," IEEE transactions on power systems, vol. 31, no. 3, pp. 1963-1971, 2015.

[10] H. Hao, B. M. Sanandaji, K. Poolla, and T. L. Vincent, "Aggregate flexibility of thermostatically controlled loads," IEEE Transactions on Power Systems, vol. 30, no. 1, pp. 189-198, 2014.

[11] P. Aristidou, S. Lebeau, and T. Van Cutsem, "Power system dynamic simulations using a parallel two-level schur-complement decomposition," IEEE Transactions on Power Systems, vol. 31, pp. 3984-3995, Sep. 2016.

[12] F. Escobar, J. García, J. Víquez, G. Valverde, and P. Aristidou, "A combined high-, medium-, and low-voltage test system for stability studies with DERs," in Proc. of the 2020 PSCC, Porto, June 2020. 